\title{
EFEITO DO HIDRORRESFRIAMENTO NA QUALIDADE DE PÊSSEGOS 'CHIMARRITA' E'CHIRIPÁ'1
}

\author{
EDUARDO SEIBERT², MICHEL ELIAS CASALI², MARCOS LAUX DE LEÃO², ERNANI PEZZI², \\ ADRIANO BRANDELLI ${ }^{3}$, RENAR JOÃO BENDER ${ }^{2}$
}

RESUMO - No presente trabalho, foram avaliados os efeitos do hidrorresfriamento para manutenção da qualidade em pêssegos 'Chimarrita' e 'Chiripá'. O hidrorresfriamento foi realizado por imersão em água com gelo a $5^{\circ} \mathrm{C}$, sendo o abaixamento da temperatura monitorado por 3 minutos. O tratamento-controle foi transferência para a câmara fria imediatamente após a colheita dos pêssegos. Os frutos foram armazenados a $0^{\circ} \mathrm{C}$ ('Chiripá') e $0,5^{\circ} \mathrm{C}$ ('Chimarrita') por $7 ; 14$ ou 21 dias e avaliados na colheita, nas saídas de frio, e mais de 2 a 3 dias a $20^{\circ} \mathrm{C}$ para o amadurecimento. $\mathrm{O}$ hidrorresfriamento foi efetivo em retirar o calor de campo dos frutos. Os frutos hidrorresfriados desidrataram mais na armazenagem, e pêssegos de ambos os tratamentos apresentaram murchamento no amadurecimento. A ocorrência de podridões não diferiu entre pêssegos hidrorresfriados e controles no armazenamento, nas duas cultivares, mas foram maiores no tratamento-controle, no amadurecimento na 'Chimarrita'. A firmeza da polpa foi igual entre os tratamentos na 'Chimarrita' e superior nos pêssegos 'Chiripá' hidrorresfriados durante a armazenagem. No amadurecimento a $20^{\circ} \mathrm{C}$, a firmeza de polpa de ambas as cultivares decresceu a valores semelhantes nos frutos-controle e nos hidrorresfriados. Após 21 dias em frio, 50\% dos pêssegos da cv. Chiripá e $15 \%$ dos pêssegos da cv. Chimarrita, de ambos os tratamentos, apresentaram lanosidade. O distúrbio retenção de firmeza ocorreu em $20 \%$ dos pêssegos 'Chiripá' e em $50 \%$ dos 'Chimarrita'.

Termos para indexação: Frutas de caroço, pré-resfriamento, frigoconservação, danos de frio.

\section{EFFECT OF HYDROCOOLING ON THE QUALITY OF 'CHIMARRITA’AND 'CHIRIPÁ’ PEACHES}

ABSTRACT - In the present work the effects of hydro cooling on the quality of 'Chimarrita' and 'Chiripá' peaches were evaluated. Peaches were hydrocooled by immersion in water at $5^{\circ} \mathrm{C}$ with flesh temperatures monitored for 3 minutes and thereafter stored at $0^{\circ} \mathrm{C}$ ('Chiripá') or $0,5^{\circ} \mathrm{C}$ ('Chimarrita') for 7,14 or 21 days. Samples were evaluated at harvest, after retrieval from cold storage and after 2 or 3 more days at $20^{\circ} \mathrm{C}$ for ripening. Hydrocooled peaches dehydrated more, but both, hydrocooled and control peaches showed symptoms of shriveling after ripening at $20^{\circ} \mathrm{C}$. Decay losses of both cultivars did not differ amongst treatments during storage, though, were higher in 'Chimarrita' control peaches after ripening at $20^{\circ} \mathrm{C}$. Flesh firmness was similar in hydrocooled and not hydrocooled 'Chimarrita' peaches, but was higher in hydrocooled 'Chiripá' peaches during storage. In both cultivars flesh firmness decreased to the same values after ripening at $20^{\circ} \mathrm{C}$. After 21 days of cold storage, woolliness was observed in $50 \%$ of 'Chiripá' and in $15 \%$ of 'Chimarrita' hydro cooled and not hydrocooled peaches. Firmness retention was observed in $20 \%$ of 'Chiripá' and in 50\% of 'Chimarrita' peaches.

Index terms: Stone fruits, pre cooling, cold storage, chilling injuries.

\section{INTRODUÇÃO}

A cultura do pessegueiro apresenta grande importância para a região Sul do Brasil, responsável por aproximadamente $50 \%$ da produção brasileira desta fruta (Marodin \& Sartori, 2000). Como os pêssegos são produtos altamente perecíveis, após a colheita, devido ao aumento da sua taxa respiratória, devem ser rapidamente resfriados para manter a qualidade de um produto fresco. Uma forma de se alcançar este objetivo mais rapidamente é realizando o pré-resfriamento dos frutos logo após a colheita, antes de serem colocados em armazenagem refrigerada. O préresfriamento é uma técnica que permite remover o calor com que os frutos vêm do pomar após a colheita, reduzindo a temperatura para valores próximos a $0^{\circ} \mathrm{C}$, a fim de se reduzirem perdas de se e controlarem doenças e outras deteriorações (Bennett \& Wells, 1976).
Vários são os métodos para alcançar o pré-resfriamento de produtos agrícolas, como o hidrorresfriamento, o resfriamento por ar forçado ou o resfriamento a vácuo. O hidrorresfriamento é o método de pré-resfriamento em que o meio refrigerante é a água, podendo ser feito por imersão ou por aspersão. Quando feito por imersão, os frutos são completamente submersos em água. O préresfriamento em água é usado para culturas como maçãs e mangas. Para pêssegos, no entanto, essa técnica é pouco utilizada no Brasil. Uma das razões para evitar seu uso comercial em pêssegos no Brasil, é que a cultura do pessegueiro apresenta um grande problema que são as doenças causadas por fungos, como a podridão-parda (Monilinia fructicola). Com o hidrorresfriamento, o problema poderia aumentar, já que inóculo do fungo poderia ser disseminado através da água usada no pré-resfriamento, contaminando os frutos e aumentando a ocorrência de podridões durante a armazenagem, como observado por Kluch et al. (2003),

\footnotetext{
(Trabalho 166-06). Recebido em : 30-10-2006. Aceito para publicação em: 16-03-2007.

${ }^{2}$ UFRGS, Laboratório de Fisiologia Pós-Colheita, Depto de Horticultura e Silvicultura, Faculdade de Agronomia, Caixa Postal 15.100, Porto Alegre-RS. eduseibert@ig.com.br, casali@roulier.com.br, lauxleao@yahoo.com.br, pezzi@ufrgs.br, rjbe@vortex.ufrgs.br

3 ICTA/UFRGS, Laboratório de Bioquímica dos Alimentos. abrand@ufrgs.br
} 
em pêssegos 'Chiripá' pré-resfriados em água.

Uma preocupação adicional é a ocorrência de danos por frio. Como os pêssegos são suscetíveis aos danos por frio, há a possibilidade de o pré-resfriamento aumentar a ocorrência e a intensidade destes danos, como a lanosidade. A preocupação com o aumento de desordens fisiológicas em produtos submetidos ao pré-resfriamento é citada por DeEll et al. (2000) e por Lallu et al. (2000), que testaram o hidrorresfriamento em chuchu e aspargos, respectivamente. Apesar desta possibilidade, Kluch et al. (2003) e Brackmann et al. (2001) não observaram aumento na manifestação de danos por frio em pêssegos hidrorresfriados.

Com essas considerações, o objetivo do trabalho foi avaliar o efeito do rápido pré-resfriamento em água por imersão sobre a qualidade dos frutos e a ocorrência de danos por frio em pêssegos das cultivares Chimarrita e Chiripá, ao longo do armazenamento refrigerado e do amadurecimento.

\section{MATERIAL E MÉTODOS}

Pêssegos 'Chimarrita' foram colhidos em dezembro de 2001, em um pomar comercial em Charqueadas-RS, e pêssegos 'Chiripá' foram colhidos em janeiro de 2002, em um pomar comercial em Farroupilha, RS. Os pêssegos foram colhidos no estádio de maturação semimaduro, com início da mudança de coloração de fundo da epiderme de verde para branco-creme e abundante coloração de cobrimento. Logo após a colheita, os pêssegos foram selecionados e submetidos ao hidrorresfriamento por imersão.

$\mathrm{O}$ hidrorresfriamento foi realizado por imersão dos frutos em água contendo gelo em cubos, à temperatura de $5^{\circ} \mathrm{C}$, usando tanques de $500 \mathrm{~L}$ preenchidos com água até $2 / 3$ de sua capacidade. Após a estabilização da temperatura da água, 120 frutos foram completamente submersos por vez, e o abaixamento da temperatura, monitorado por 3 minutos. Essa operação foi repetida quatro vezes até todos os frutos destinados ao hidrorresfriamento serem resfriados. Durante o pré-resfriamento, foi realizada a leitura da temperatura da polpa de alguns frutos através de sensores de temperatura do tipo PT100 conectados a um sistema de aquisição de dados. Os frutos-controle, sem pré-resfriamento, foram armazenados diretamente em frio, sendo o abaixamento da temperatura monitorado dentro da câmara fria, por 45 minutos, também por sensores tipo PT100 conectados a um sistema de aquisição de dados.

Após o hidrorresfriamento, os frutos foram embalados em caixas plásticas de $10 \mathrm{~kg}$ de capacidade e armazenados sob refrigeração. Os pêssegos 'Chimarrita' foram armazenados em Charqueadas, em uma câmara fria de produtor, a $0,5^{\circ} \mathrm{C}$ e $90 \%$ de umidade relativa (UR), por 7; 14 ou 21 dias. Os pêssegos 'Chiripá' foram armazenados em Farroupilha (unidade de armazenagem da Prefeitura Municipal), em uma câmara a $0^{\circ} \mathrm{C} \mathrm{e} 90 \%$ de UR, por 7; 14 ou 21 dias. $\mathrm{Na}$ instalação do experimento, nas saídas da armazenagem refrigerada e depois de mais 2 a 3 dias a $20^{\circ} \mathrm{C}$ para amadurecimento, amostras foram avaliadas no Laboratório de Horticultura da Faculdade de Agronomia da UFRGS. As variáveis analisadas foram: perdas de massa (\%), medidas por diferença de peso entre a instalação do experimento e cada avaliação após a armazenagem refrigerada e amadurecimento; ocorrência de podridões (\%), avaliada em lesões maiores que $0,5 \mathrm{~cm}$ e com sintomas de ataques por patógenos; cor vermelha na epiderme $(\%)$, avaliada visualmente; firmeza da polpa $(\mathrm{N})$, medida em dois lados diametralmente opostos dos frutos com penetrômetro manual equipado com ponteira Magness-Taylor de 7,9mm diâmetro; sólidos solúveis totais (SST), medidos com um refratômetro de bancada ( ${ }^{\circ}$ Brix); acidez total titulável (AT), por titulação com solução de $\mathrm{NaOH} 0,1 \mathrm{~N}$ até pH 8,1 (\% ac. málico).

Os danos por frio (\%) foram avaliados visualmente após cortar os frutos em duas metades, para determinar a presença de lanosidade, escurecimento da polpa e retenção de firmeza. O escurecimento foi determinado observando a coloração da polpa para classificar os frutos de acordo com a seguinte escala: $1=$ sadios (polpa sem escurecimento); 2 = leve escurecimento (escurecimento em $0-25 \%$ da polpa); $3=$ moderado escurecimento (25-50\% da polpa com escurecimento); $4=$ severo escurecimento ( $>50 \%$ da polpa com escurecimento). Frutos com a polpa com textura corticosa e firmeza superior a $40 \mathrm{~N}$ foram classificados como apresentando o distúrbio fisiológico denominado retenção de firmeza. Apertando uma metade dos pêssegos com a mão, foram determinadas intensidades de lanosidade de acordo com a escala: 1 = sadios (abundante liberação de suco); 2 = lanosidade leve (moderada liberação de suco); 3 = moderada lanosidade (baixa liberação de suco); 4 = severa lanosidade (sem suco).

Pêssegos suculentos, sem sinais de escurecimento da polpa, lanosidade e retenção de firmeza foram considerados sadios e aptos para comercialização.

O delineamento experimental foi completamente casualizado, com quatro repetições de 15 frutos por unidade experimental e data de avaliação e tratamento. O programa de análise estatística SANEST (Zonta \& Machado, 1986) foi usado para fazer a análise de variância. Para comparar médias entre os tratamentos, foi usado o teste de Tukey $(P<0,05)$.

\section{RESULTADOS E DISCUSSÃO}

A temperatura média da polpa dos pêssegos 'Chiripá' no momento do início do tratamento foi de $24,5^{\circ} \mathrm{C}$. Com o hidrorresfriamento, houve uma diminuição média de $14,2^{\circ} \mathrm{C}$ durante os 3 minutos de pré-resfriamento com água na temperatura de $5^{\circ} \mathrm{C}$. Nos frutos-controle, armazenados diretamente na câmara fria, houve um abaixamento de $8^{\circ} \mathrm{C}$ na temperatura da polpa dos frutos durante os 45 minutos de monitoramento. Já com pêssegos 'Chimarrita' a temperatura dos frutos no início do pré-resfriamento foi de $23,4^{\circ} \mathrm{C}$ e, nos 3 minutos do hidrorresfriamento, diminuiu em $12,5^{\circ} \mathrm{C}$. Nos pêssegos do tratamento-controle da cv. Chimarrita, a temperatura da polpa diminuiu apenas $5,1^{\circ} \mathrm{C}$ durante os 45 minutos de monitoramento. Os resultados indicam que o hidrorresfriamento foi efetivo em retirar o calor de campo dos frutos.

Os pêssegos submetidos ao hidrorresfriamento desidrataram mais que os controles durante a armazenagem, sendo isso visualizado na 'Chimarrita' após 14 e 21 dias em frio, e na 'Chiripá' somente após 21 dias a $0^{\circ} \mathrm{C}$ (Tabela 1). Durante o amadurecimento, houve um aumento considerável na perda de massa que foi igual entre os tratamentos na 'Chimarrita', enquanto na 'Chiripá' a desidratação média foi maior nos pêssegos 
hidroresfriados, mas sem diferenças em cada data de avaliação. No amadurecimento, os pêssegos 'Chimarrita' após os 7 e os 21 dias a $0,5^{\circ} \mathrm{C}$, e os pêssegos 'Chiripá' após os 7 e os 14 dias a $0^{\circ} \mathrm{C}$ apresentaram alguns frutos com enrugamento visível principalmente na região dos ombros dos frutos. Os valores de perda de massa na maturação são uma conseqüência do avançado estádio de amadurecimento dos frutos devido ao tempo em que permaneceram a $20^{\circ} \mathrm{C}$, levando a uma natural desidratação dos frutos. Para Akbudak \& Eris (2004), esse é um dos principais problemas para a armazenagem de pêssegos por períodos prolongados, visto que altas desidratações causam má aparência aos frutos.

Um dos argumentos desfavoráveis, além da disseminação de inóculo à recomendação do pré-resfriamento em água para frutos de caroço, é a possibilidade de haver danos à epiderme e, com isso, haver uma propensão a maior murchamento dos frutos. Contudo, se os resultados do presente experimento não indicam claramente esse fato, as diferenças significativas não corroboram este argumento.

A ocorrência de podridões não apresentou diferenças significativas entre os pêssegos pré-resfriados e os pêssegos do tratamento-controle na cultivar Chiripá, tanto na saída da armazenagem refrigerada como após o amadurecimento a $20^{\circ} \mathrm{C}$. Nos pêssegos da cultivar Chimarrita, durante o período de armazenagem também não foram significativas as diferenças em percentual de frutos com podridão (Tabela 1). No entanto, após o período de amadurecimento a $20^{\circ} \mathrm{C}$, os pêssegos desta cultivar que não foram pré-resfriados em água, apresentaram percentuais de frutos podres significativamente maiores após 7 e 14 dias a $0,5^{\circ} \mathrm{C}$, o que resultou em maior média de perdas de frutos no tratamento-controle. Essa maior perda por podridões nos frutoscontrole indica que as podridões não foram devidas ao préresfriamento em água, contrariando resultados de Kluch et al. (2003) e Brackmann et al. (2001), que observaram maior ocorrência de podridões em pêssegos hidrorresfriados. Segundo Brackmann et al. (1994), o pré-resfriamento em água possibilita a contaminação dos frutos com inóculo presente presentes na água do préresfriador.

Os valores de SST foram estatisticamente maiores nos pêssegos 'Chimarrita' pré-resfriados na saída da câmara e após a exposição a $20^{\circ} \mathrm{C}$. Nos pêssegos 'Chiripá', foram determinados maiores teores de SST apenas na saída da armazenagem refrigerada. Segundo Brackmann et al. (1994), o hidrorresfriamento pela mais rápida retirada do calor de campo pode diminuir o processo de degradação dos açúcares e ácidos devido à diminuição da velocidade do metabolismo após a colheita. Von Mollendorf et al., já em 1992, concluíram pelos efeitos positivos do préresfriamento na qualidade de frutas de caroço. As maiores médias de SST nos frutos hidrorresfriados também podem ser devidas às maiores perdas de umidade verificadas nestes frutos. De igual modo, podem ter sido influenciados os teores de acidez total titulável (Tabela 2) de ambas as cultivares. As diferenças no entanto foram bastante reduzidas, mesmo que significativas, como no caso dos pêssegos da cultivar Chiripá hidrorresfriados que foram menos ácidos que os controles na avaliação na saída da armazenagem refrigerada.
Os pêssegos 'Chimarrita' hidrorresfriados, na maior parte das análises, não apresentaram diferenças de firmeza para os frutoscontrole, comportamento observado na conservação em frio e no amadurecimento a $20^{\circ} \mathrm{C}$ (Figura 1). A firmeza inicial foi semelhante nos dois tratamentos de pêssegos 'Chimarrita' indicando que a aleatorização das amostras foi adequada. A firmeza, na instalação do experimento, próxima a $64 \mathrm{~N}$, decresceu desde a colheita até os 14 dias de armazenagem. Aos 21 dias, determinou-se um aumento significativo de firmeza em comparação com as análises anteriores (Figura 1A). Durante o amadurecimento, já na análise após 7 dias a $0,5^{\circ} \mathrm{C}$, a firmeza baixou a valores próximos a 5,0N em ambos os tratamentos (Figura 1B), comportamento que se manteve nas demais avaliações. A baixa firmeza foi devida ao tempo no qual os frutos foram mantidos a $20^{\circ} \mathrm{C}$ antes de sua análise, que foi de 2 a 3 dias.

Nos pêssegos 'Chiripá', a firmeza da polpa diminuiu constantemente nos dois tratamentos, durante a armazenagem. Os frutos pré-resfriados foram significativamente mais firmes que os pêssegos que não foram hidrorresfriados aos 7 e 14 dias a $0^{\circ} \mathrm{C}$ (Figura 1C). No amadurecimento a $20^{\circ} \mathrm{C}$, assim como ocorrido na 'Chimarrita', a firmeza decresceu a valores semelhantes nos dois tratamentos, com uma média inferior a $10 \mathrm{~N}$ e sem diferenças significativas (Figura 1D). Brackmann et al. (2000) também observaram uma firmeza superior em pêssegos 'Chiripá' hidrorresfriados durante a armazenagem. Esses resultados observados com as cultivares Chimarrita e Chiripá destacam também que o controle do metabolismo das paredes celulares não é suficientemente inibido com uso do frio e que devem ser avaliados outros métodos para bloquear a queda acentuada de firmeza após a armazenagem refrigerada.

Nas duas cultivares, no amadurecimento a partir dos 7 dias em frio, seguidos de 2 a 3 dias a $20^{\circ} \mathrm{C}$, houve sempre um mínimo de $50 \%$ de frutos sobremaduros com firmeza inferior a $9 \mathrm{~N}$ (Figura 1B e 1D). Essta firmeza não inviabiliza o consumo; porém, para a comercialização de pêssegos com esse amolecimento de tecidos, é necessário um manuseio muito cuidadoso para evitar danos mecânicos durante o transporte e a comercialização. Esses dados indicam que pêssegos que estavam em armazenagem refrigerada, devem ser manuseados com cuidado e rapidamente comercializados, quando isso ocorrer sob condições de altas temperaturas.

Os pêssegos 'Chimarrita' e 'Chiripá' mostraram suscetibilidade à lanosidade e à retenção de firmeza da polpa, mas não foram observados sintomas de escurecimento da polpa. Os danos de frio ocorreram nas duas cultivares após os 21 dias de armazenagem refrigerada e não foram observadas diferenças significativas entre os frutos do tratamento-controle e os hidrorresfriados quanto à lanosidade (Tabela 3 ). O percentual de pêssegos 'Chiripá' com lanosidade nesta data foi alto, próximo a $70 \%$ nos dois tratamentos, sendo a intensidade do dano moderada a severa. $\mathrm{Na}$ 'Chimarrita', a porcentagem de frutos que manifestaram lanosidade, foi menor, sendo a intensidade do dano moderada.

Uma alta porcentagem de pêssegos 'Chimarrita' manifestou sintomas de retenção de firmeza, alcançando um mínimo de $50 \%$ dos frutos-controle e hidrorresfriados. $\mathrm{Na}$ 
'Chiripá', um número menor de frutos manifestou o dano sendo este significativamente menor nos frutos hidrorresfriados. $\mathrm{Na}$ cultivar Chimarrita, além da alta firmeza aos 21 dias de armazenagem refrigerada, superior a $40 \mathrm{~N}$, os pêssegos apresentaram a polpa com textura emborrachada. Segundo Luchsinger (2000), a $0^{\circ} \mathrm{C}$, produz-se uma forte inibição do amadurecimento, devido à baixa taxa de produção de etileno com uma retenção da firmeza da polpa, atrasando a visualização dos sintomas de lanosidade. Ju et al. (2000), no entanto, citam que lanosidade e retenção de firmeza são diferentes desordens causadas pela exposição ao frio, e que causam a polpa seca nos frutos. Essas anomalias na firmeza de pêssegos que sofreram danos de frio também foram observadas por Fernández-Trujillo et al. (1998).

A lanosidade no amadurecimento foi observada em menor porcentagem de frutos, não superior a $15 \%$ nas duas cultivares, após 21 dias em frio, com intensidade baixa do dano. Uma explicação para a baixa visualização do dano no amadurecimento é a possibilidade da ocorrência do fenômeno reversão da lanosidade. Segundo Luchsinger (2000), os sintomas de lanosidade podem sofrer reversão durante o período de amadurecimento após a armazenagem refrigerada, o que geralmente ocorre após 2 dias a $20^{\circ} \mathrm{C}$. Como os pêssegos 'Chimarrita' e 'Chiripá' foram analisados no $2^{\circ}$ e $3^{\circ}$ dias a $20^{\circ} \mathrm{C}$, é possível que a lanosidade tenha sofrido .

TABELA 1 - Perda de massa fresca e incidência de podridões observadas após até 21 dias em armazenagem refrigerada a $0,5^{\circ} \mathrm{C}$ (cv. Chimarrita) ou $0^{\circ} \mathrm{C}$ (cv.Chiripá) a $90 \%$ de UR e mais 2 a 3 dias de amadurecimento a $20^{\circ} \mathrm{C}$, em pêssegos (cv.Chimarrita) e (cv.Chiripá), submetidos ao hidrorresfriamento rápido (HR). Controle (C).Valores em percentual (\%).

\begin{tabular}{|c|c|c|c|c|c|c|c|c|c|}
\hline \multicolumn{10}{|c|}{ Chimarrita } \\
\hline \multirow{3}{*}{$\begin{array}{c}\text { Dias a } \\
0,5^{\circ} \mathrm{C}\end{array}$} & \multicolumn{4}{|c|}{ Armazenagem } & \multirow{3}{*}{$\begin{array}{c}\text { Dias a } \\
0,5^{\circ}+20^{\circ} \\
\text { C }\end{array}$} & \multicolumn{4}{|c|}{ Amadurecimento } \\
\hline & \multicolumn{2}{|c|}{ Perda de peso } & \multicolumn{2}{|c|}{ Podridões } & & \multicolumn{2}{|c|}{ Perda de peso } & \multicolumn{2}{|c|}{ Podridões } \\
\hline & $\mathbf{C}$ & HR & $\mathbf{C}$ & HR & & $\mathbf{C}$ & HR & $\mathbf{C}$ & HR \\
\hline $\mathbf{0}$ & --- & --- & --- & --- & $\mathbf{0}+3$ & $6,9 \mathrm{a}$ & $6,9 \mathrm{a}$ & $0,0 \mathrm{a}$ & $0,0 \mathrm{a}$ \\
\hline 7 & $0,0 \mathrm{a}$ & $0,5 \mathrm{a}$ & $0,0 \mathrm{a}$ & $0,0 \mathrm{a}$ & $7+3$ & $13,0 \mathrm{a}$ & $13,2 \mathrm{a}$ & $70,5 \mathrm{a}$ & $33,8 \mathrm{~b}$ \\
\hline 14 & $1,7 \mathrm{~b}$ & $3,7 \mathrm{a}$ & $4,7 \mathrm{a}$ & $3,1 \mathrm{a}$ & $14+2$ & $8,1 \mathrm{a}$ & $8,3 \mathrm{a}$ & $49,4 \mathrm{a}$ & $16,7 \mathrm{~b}$ \\
\hline 21 & $3,5 \mathrm{~b}$ & $7,1 \mathrm{a}$ & $8,6 \mathrm{a}$ & $9,4 \mathrm{a}$ & $21+2$ & $10,3 \mathrm{a}$ & $9,3 \mathrm{a}$ & $25,8 \mathrm{a}$ & $21,6 \mathrm{a}$ \\
\hline Média & $1,7 \mathrm{~b}$ & $3,8 \mathrm{a}$ & $4,4 \mathrm{a}$ & $4,2 \mathrm{a}$ & Média & $9,6 \mathrm{a}$ & $9,4 \mathrm{a}$ & $36,4 \mathrm{a}$ & $18,0 \mathrm{~b}$ \\
\hline CV (\%) & 18,5 & & 90,1 & & CV (\%) & 11,9 & & 52,0 & \\
\hline
\end{tabular}

\begin{tabular}{|c|c|c|c|c|c|c|c|c|c|}
\hline \multicolumn{10}{|c|}{ Chiripá } \\
\hline \multirow{3}{*}{$\begin{array}{c}\text { Dias a } \\
0^{\circ} \mathrm{C}\end{array}$} & \multicolumn{4}{|c|}{ Armazenagem } & \multirow{3}{*}{$\begin{array}{c}\text { Dias a } \\
0^{\circ}+20^{\circ} \mathrm{C}\end{array}$} & \multicolumn{4}{|c|}{ Amadurecimento } \\
\hline & \multicolumn{2}{|c|}{ Perda de peso } & \multicolumn{2}{|c|}{ Podridões } & & \multicolumn{2}{|c|}{ Perda de peso } & \multicolumn{2}{|c|}{ Podridões } \\
\hline & $\mathbf{C}$ & HR & $\mathbf{C}$ & HR & & $\mathbf{C}$ & HR & $\mathbf{C}$ & HR \\
\hline $\mathbf{0}$ & $--^{1}$ & --- & --- & --- & $\mathbf{0}+3$ & $5,3 \mathrm{a}$ & $6,0 \mathrm{a}$ & $0,0 \mathrm{a}$ & $0,0 \mathrm{a}$ \\
\hline 7 & $1,0 \mathrm{a}^{2}$ & $1,7 \mathrm{a}$ & $0,0 \mathrm{a}$ & $0,8 \mathrm{a}$ & $7+3$ & $5,0 \mathrm{a}$ & $8,0 \mathrm{a}$ & $5,4 \mathrm{a}$ & $8,3 \mathrm{a}$ \\
\hline 14 & $1,1 \mathrm{a}$ & $1,5 \mathrm{a}$ & $2,7 \mathrm{a}$ & $7,3 \mathrm{a}$ & $14+3$ & $5,3 \mathrm{a}$ & $7,9 \mathrm{a}$ & $21,4 \mathrm{a}$ & $12,5 \mathrm{a}$ \\
\hline 21 & $2,4 \mathrm{~b}$ & $4,7 \mathrm{a}$ & $7,3 \mathrm{a}$ & $10,4 \mathrm{a}$ & $21+3$ & $4,3 \mathrm{a}$ & $6,5 \mathrm{a}$ & $10,7 \mathrm{a}$ & $15,9 \mathrm{a}$ \\
\hline Média & $1,5 \mathrm{~b}$ & $2,6 \mathrm{a}$ & $3,3 \mathrm{a}$ & $6,2 \mathrm{a}$ & Média & $5,0 \mathrm{~b}$ & $7,1 \mathrm{a}$ & $9,4 \mathrm{a}$ & $9,2 \mathrm{a}$ \\
\hline CV (\%) & & 29,0 & 95,9 & & CV (\%) & & 17,8 & 111,7 & \\
\hline
\end{tabular}

${ }^{1}$ (-) Indicam que o parâmetro não foi analisado neste ano).

${ }^{2}$ Médias seguidas da mesma letra na linha, dentro de cada parâmetro e período de avaliação (armazenagem e amadurecimento), não diferem entre si, pelo teste de Tukey $(P<0,05)$. 
TABELA 2 - Teores de sólidos solúveis totais (SST) e acidez total titulável (AT) na colheita, após 21 dias em armazenagem refrigerada a $0,5^{\circ} \mathrm{C}$ (Chimarrita) ou $0^{\circ} \mathrm{C}$ (Chiripá) e $90 \%$ de UR e mais 2 a 3 dias de amadurecimento a $20^{\circ} \mathrm{C}$, em pêssegos (cv.Chimarrita) e (cv.Chiripá), submetidos ao hidrorresfriamento rápido (HR) e controle (C)..

\begin{tabular}{|c|c|c|c|c|c|}
\hline \multirow{2}{*}{ Parâmetros } & & \multicolumn{2}{|l|}{ Chimarrita } & \multicolumn{2}{|l|}{ Chiripá } \\
\hline & & Controle & HR & Controle & HR \\
\hline \multirow[t]{3}{*}{ SST ( ${ }^{\circ}$ Brix) } & Colheita & $13,9 \mathrm{a}^{1}$ & $13,8 \mathrm{a}$ & $12,0 \mathrm{a}$ & $12,6 \mathrm{a}$ \\
\hline & 21 dias & $13,0 \mathrm{~b}$ & $13,6 \mathrm{a}$ & $11,8 \mathrm{~b}$ & $12,8 \mathrm{a}$ \\
\hline & +2 a 3 dias & $13,8 \mathrm{~b}$ & $14,2 \mathrm{a}$ & $13,1 \mathrm{a}$ & $13,1 \mathrm{a}$ \\
\hline \multirow[t]{3}{*}{ AT (\% ác. málico) } & Colheita & $0,27 \mathrm{a}$ & $0,25 \mathrm{a}$ & $0,41 \mathrm{a}$ & $0,32 \mathrm{~b}$ \\
\hline & 21 dias & $0,28 \mathrm{a}$ & $0,27 \mathrm{a}$ & $0,36 \mathrm{a}$ & $0,32 \mathrm{~b}$ \\
\hline & +2 a 3 dias & $0,36 \mathrm{a}$ & $0,36 \mathrm{a}$ & $0,38 \mathrm{a}$ & 0,38 \\
\hline
\end{tabular}

${ }^{1}$ Médias seguidas da mesma letra na linha, dentro de cada parâmetro e período de avaliação (armazenagem e amadurecimento), não diferem entre si , pelo teste de Tukey $(P<0,05)$.


FIGURA 1 - Firmeza da polpa após até 21 de armazenagem refrigerada a $0,5^{\circ} \mathrm{C}$ (cv.Chimarrita) ou $0^{\circ} \mathrm{C}$ (cv.Chiripá) e $90 \%$ de UR e mais 2 a 3 dias a $20^{\circ} \mathrm{C}$ para amadurecimento de pêssegos (cv.Chimarrita) (A e B) e (cv.Chiripá) (C e D) submetidos ao hidrorrefriamento. Barra vertical indica diferença mínima significativa $(P<0,05)$.

TABELA 3 - Danos por frio após até 21 dias em armazenagem refrigerada a $0,5^{\circ} \mathrm{C}$ (cv.Chimarrita) ou $0^{\circ} \mathrm{C}$ (cv.Chiripá) e $90 \%$ de UR e mais 2 a 3 dias de amadurecimento a $20^{\circ} \mathrm{C}$, em pêssegos (cv.Chimarrita) e (cv.Chiripá), submetidos ao hidrorresfriamento (HR).

\begin{tabular}{c|cccccccc}
\hline & \multicolumn{3}{c}{ Chimarrita } & \multicolumn{3}{c}{ Chiripá } \\
\hline \multirow{2}{*}{ Dias } & Lanosidade & \multicolumn{3}{c}{ Retenção de firmeza } & Lanosidade & Retenção de firmeza \\
\cline { 2 - 8 } & Controle & HR & Controle & HR & Controle & HR & Controle & HR \\
\hline 7 & $0 \mathrm{a}$ & $0 \mathrm{a}$ & $0 \mathrm{a}$ & $0 \mathrm{a}$ & $0 \mathrm{a}$ & $0 \mathrm{a}$ & $0 \mathrm{a}$ & $0 \mathrm{a}$ \\
14 & $0 \mathrm{a}$ & $0 \mathrm{a}$ & $0 \mathrm{a}$ & $0 \mathrm{a}$ & $0 \mathrm{a}$ & $0 \mathrm{a}$ & $0 \mathrm{a}$ & $0 \mathrm{a}$ \\
21 & $16,7 \mathrm{a}$ & $8,3 \mathrm{a}$ & $50 \mathrm{a}$ & $58 \mathrm{a}$ & $75 \mathrm{a}$ & $67 \mathrm{a}$ & $16,3 \mathrm{a}$ & $3,3 \mathrm{~b}$ \\
Média & $5,6 \mathrm{a}$ & $2,8 \mathrm{a}$ & $19,5 \mathrm{a}$ & $16,7 \mathrm{a}$ & $25 \mathrm{a}$ & $22 \mathrm{a}$ & $5,2 \mathrm{a}$ & $1,1 \mathrm{~b}$ \\
\hline CV(\%) & 67,6 & & 85,0 & & 59,4 & & 102,1 &
\end{tabular}

Médias seguidas da mesma letra na linha, dentro de cada parâmetro e período de avaliação (armazenagem e amadurecimento), não diferem entre si, pelo teste de Tukey $(P<0,05)$.

\section{CONCLUSÕES}

1- O resfriamento rápido em água de pêssegos das cultivares Chimarrita e Chiripá é uma prática possível de uso comercial porque retira rapidamente o calor de campo dos frutos e não implica maior ocorrência de podridões durante a armazenagem dos pêssegos, mas favorece maiores perdas de massa.
2- O resfriamento rápido em água tem efeitos limitados sobre a manutenção da qualidade dos pêssegos durante a armazenagem e não previne a manifestação de danos de frio nas cultivares Chimarrita e Chiripá, que são suscetíveis aos danos por frio após 21 de armazenagem refrigerada.

3- Os danos de frio mais importantes nas cultivares Chimarrita e Chiripá são a lanosidade e a retenção de firmeza. 


\section{REFERÊNCIAS}

AKBUDAK, B.; ERIS, A. Physical and chemical changes in peaches and nectarines during the modified atmosphere storage. Food Control, Oxford, v.15, n.4, p-307-313, 2004.

BENNETT, A.H.; WELLS, J.M. Hydraircooling: a new precooling method with special applicattion for waxed peaches. Journal of the American Society for Horticultural Science, Mount Vernon, n.101, v.4, p.428-431, 1976.

BRACKMANN, A.; MAZARO, S.M.; CECCHINI, R. Efeito do pré-resfriamento e do tratamento pós-colheita sobre a qualidade de maçãs, cv. Golden Delicious e Fuji, durante o armazenamento em atmosfera normal e controlada. Revista Brasileira de Fruticultura, Cruz das Almas, v.16, n.1, p.7-14, 1994.

BRACKMANN, A.; CERETTA, M; WACLAWOSKY, A.J. Préresfriamento e tratamento pós-colheita de pêssegos cv. Chiripá frigoconservados. Revista Brasileira de Agrociência, Pelotas, v.6, n.1, p.27-29, 2000.

BRACKMANN, A.; HUNSCHE, M.; CERETTA, M. Préresfriamento e absorção de etileno durante $\mathrm{o}$ armazenamento de pêssegos cv. Chiripá. Revista Brasileira de Armazenamento, Viçosa, v.26, n.1, p.12-15, 2001.

DeELL, J.R.; VIGNEAULT, C.; LEMERRE, S. Water temperature for hydrocooling field cucumbers in relation to chilling injury during storage. Postharvest Biology and Technology, Amsterdam, v.18, n.2, p.27-32, 2000.

FERNÁNDEZ-TRUJILLO, J.P.; CANO, A.; ARTÉS, F. Physiological changes in peaches related to chilling injury and ripening. Postharvest Biology and Technology, Amsterdam, v.13, p.109-119, 1998.

JU, Z.; DUAN, Y.; JU, Z. Leatheriness and mealiness of peaches in relation to fruit maturity and storage temperature. Journal of Horticultural Science \& Biotechnology, Ashford, v.75, n.1, p.86-91, 2000.

KLUCH, H.D.W.; MELlO, A.M. de; FREITAS, S.T.; BRACKMANN, A. Efeito do pré-resfriamento e condições de armazenamento sobre a qualidade físico-química e lanosidade de pêssegos cv. Chiripá. Revista Brasileira de Agrociência, Pelotas, v.9, n.3, p.269-272, 2003.

LALLU, N.; YEARSLEY, C.W.; ELGAR, H.J. Effects of cooling treatments and physical damage on tip rot and postharvest quality of asparagus spears. New Zealand Journal of Crop and Horticultural Science, Wellington, v.28, p.27-36, 2000.

LUCHSINGER, L. 2000. Avanços na conservação de frutas de caroço. In: SIMPÓSIO INTERNACIONAL DE FRUTAS DE CAROÇO, PÊSSEGOS, NECTARINAS EAMEIXAS, 1., 2000, Porto Alegre. Anais... p.95-105.

MARODIN, G.A.B.; SARTORI, I.A. 2000. Situação das frutas de caroço no Brasil e no Mundo. In: SIMMPÓSIO INTERNACIONAL DE FRUTAS DE CAROÇO, PÊSSEGOS, NECTARINAS E AMEIXAS, 1., 2000, Porto Alegre. Anais... p.7-16

VONMOLLENDORFF,L.J.;JACOBS, G.; DE VILLIERS, O.T.Cold storage influences internal characteristics of nectarines during ripening. HortScience, Alexandria, v.27, n.12, p.1295-
$1297,1992$.

ZONTA, E.P.; MACHADO, A.A. Sistema de análise estatística para microcomputadores - "SANEST" (software). Pelotas: UFPEL, Instituto de Física e Matemática, 1986. 